\title{
HPV16 E7-specific HLA-A*02:01-restricted IgG1-Fc Fusion Protein CUE-101
}

National Cancer Institute

\section{Source}

National Cancer Institute. HPV16 E7-specific HLA-A*02:01-restricted Ig G1-FC Fusion

Protein CUE-101. NCI Thesaurus. Code C162459.

A fusion protein composed of a human leukocyte antigen (HLA) complex, HLA-A*0201, with a peptide epitope derived from the human papillomavirus type 16 (HPV16) E7 protein (amino acid residues 11-20), a reduced affinity human interleukin-2 (IL-2) variant, and an effector attenuated human immunog lobulin G1 (IgG1) Fc domain, with potential antineoplastic and immunostimulatory activities. Upon administration, CUE-101 targets and selectively binds to E7-specific CD8-positive T-cells present in patients with HPV16driven malignancies. This may induce the secretion of inflammatory cytokines such as interferon gamma (IFN gamma) and promote the activation and expansion of tumorspecific CD8-positive cells, which may lead to T-cell-mediated elimination of tumor cells expressing the HPV16 E7 antigen. The HPV16 E7 oncoprotein is a cell surface glycoprotein and tumor-associated antigen (TAA) that is overexpressed in various HPVassociated cancers. 